\title{
Concentration Effects on the Thermophoresis of Aerosol Spheres
}

\author{
Huan J. Keh ${ }^{1}$ and Na Y. Ho \\ Department of Chemical Engineering, National Taiwan University, Taipei 106-17, Taiwan, Republic of China
}

Received February 4, 1999; accepted April 27, 1999

\begin{abstract}
The thermophoretic motion of a homogeneous suspension of identical spherical particles of arbitrary thermal conductivity and surface properties is considered under conditions of small Knudsen, Peclet, and R eynolds numbers. The effects of interaction of the individual particles are taken into explicit account by employing a unit cell model which is known to provide good predictions for the sedimentation of monodisperse suspensions of spherical particles. The appropriate equations of conservation of energy and momentum are solved for each cell, in which a spherical particle is envisaged to be surrounded by a concentric shell of suspending fluid, and the thermophoretic migration velocity of the particle is calculated for various cases. Analytical expressions of this mean particle velocity are obtained in closed form as functions of the volume fraction of the particles. Comparisons between the ensemble-averaged thermophoretic velocity of a test particle in a dilute suspension and our cell-model results are made. A parallel analysis for the sedimentation of aerosol spheres is also presented. $\odot 1999$ A cademic Press
\end{abstract}

Key Words: thermophoresis; sedimentation; aerosol spheres; effects of particle volume fraction; unit cell model.

\section{INTRODUCTION}

A small particle, when suspended in a gas containing a temperature gradient, experiences a force that normally drives the particle in the direction of decreasing temperature. This motion is known as thermophoresis and has been the subject of considerable study for many years (1-3). The physical explanation of this phenomenon is based on the kinetic theory of gases $(4,5)$. The higher energy molecules in the hot region of the gas impinge on the particle with greater momenta than molecules coming from the cold region, thus resulting in the migration of the particle in the direction opposite to the temperature gradient. Being a mechanism for the capture of aerosol particles on cold surfaces, thermophoresis is important in many practical applications, such as sampling of aerosol particles (6), cleaning of air (7), scale formation on surfaces of heat exchangers (8), modified chemical vapor deposition (9), microelectronic manufacturing (10), and nuclear reactor safety (11).

Based on the assumptions of small Knudsen number ( $l / a$,

${ }^{1}$ To whom correspondence should be addressed. where $a$ is the radius of the particle and $l$ is the mean free path of the gas molecules), small Peclet number ( $a U / \alpha$, where $U$ is the velocity of the particle and $\alpha$ is the thermal diffusivity of the gas), and small Reynolds number $(a U / \nu$, where $\nu$ is the kinematic viscosity of the gas), as well as the effects of temperature jump, thermal creep, and frictional slip at the gas-particle surface, Brock (12) obtained the thermophoretic velocity of an aerosol sphere in a constant temperature gradient $E_{\infty}$ as

$$
U^{(0)}=\left[\frac{2 C_{\mathrm{s}}\left(k+k_{1} C_{\mathrm{t}} l / a\right)}{\left(1+2 C_{\mathrm{m}} l / a\right)\left(2 k+k_{1}+2 k_{1} C_{\mathrm{t}} l / a\right)}\right] \frac{\eta}{\rho T_{0}} E_{\infty} .
$$

Here, $\rho, \eta$, and $k$ are the density, viscosity, and thermal conductivity, respectively, of the gas; $k_{1}$ is the thermal conductivity of the particle; $T_{0}$ is the bulk-gas absolute temperature at the particle center in the absence of the particle (or the mean gas temperature in the vicinity of the particle); $C_{\mathrm{s}}, C_{\mathrm{t}}$, and $C_{\mathrm{m}}$ are dimensionless coefficients (which are positive values) accounting for the thermal creep, temperature jump, and frictional slip phenomena, respectively, at the particle surface and must be determined experimentally for each gassolid system. Very accurate values of these slip and jump coefficients for simple gases and gas mixtures, as based on the solutions of the Boltzmann equation and arbitrary gas-solid surface conditions, have been reported (13). A set of reasonable kinetic-theory values for complete thermal and momentum accommodations appear to be $C_{\mathrm{s}}=1.17, C_{\mathrm{t}}=2.18$, and $C_{\mathrm{m}}=1.14$ (14). The Brock analysis is accurate only for a Knudsen number less than about 0.1 (so that the fluid flow is described by a continuum model), since it contains the Basset (frictional) slip correction for the drag on the particle, which departs from experiment at about this value.

In practical applications of thermophoretic motion, collections of aerosol particles are usually encountered. Thus, it is important to determine if the presence of neighboring particles significantly affects the movement of each particle. Recently, much progress has been made in the theoretical analysis concerning the applicability of Eq. [1] for two-sphere and multiple-sphere systems. Using a method of reflections, Keh and Chen (15) obtained the thermophoretic migration velocities of two spherical particles with arbitrary physical properties, arbi- 
trary size ratio, and arbitrary orientation with respect to the imposed temperature gradient correct to $O\left(r_{12}^{-7}\right)$, where $r_{12}$ is the center-to-center spacing. Another approach to investigating the two-sphere motion is to use spherical bipolar coordinates. By this means, the axisymmetric thermophoretic motion of two identical spherical particles was first examined semianalytically by Reed and Morrison (16), and later this work was extended to the situations of two arbitrary aerosol spheres (17). The third routine employed to solve this problem was the boundary collocation technique, which could be used to numerically calculate the thermophoretic velocities for a collection of two or more arbitrary spherical particles, even when they are touching one another (18).

The interaction effects between pairs of spheres can be extended to the calculation of the average thermophoretic migration velocity of particles in a relatively dilute, statistically homogeneous suspension subjected to a uniform temperature gradient. Based on the concepts of low Reynolds number hydrodynamics and statistical mechanics, Keh and Chen (15), using their reflection results for the thermal and hydrodynamic interactions between pairs of aerosol spheres, found how the average thermophoretic velocity of a dilute polydisperse suspension is affected by the volume fraction of the particles. Later, the dependence of this thermophoretic velocity on particle concentration was also determined by using more accurate information on the pair interactions for a swarm of arbitrary aerosol spheres (18). However, the effects of particle concentration on the thermophoretic mobility of a relatively dense gas-particle system have not been investigated yet.

A unit cell model has been employed successfully (and tested against the experimental data) to predict the effect of particle concentration on the mean sedimentation rate in a bounded suspension of identical spherical particles (19-21). This model involves the concept that an assemblage can be divided into a number of identical cells, one sphere occupying each cell at its center. The boundary-value problem for multiple spheres is thus reduced to the consideration of the behavior of a single sphere and its bounding envelope. Although different shapes of cells can be employed, the assumption of a spherical shape for the fictitious envelope of suspending fluid surrounding each spherical particle is of great convenience. Although the Brownian motion of small particles is not included in its analysis, the cell model is of great applicability in relatively concentrated suspensions, where the boundary effect will not be important. In this work, the cell model is used to describe the thermal and hydrodynamic interactions among aerosol spheres in a monodisperse suspension subjected to a constant temperature gradient. The analytical solutions in closed form obtained with this model enable the average thermophoretic velocity to be predicted as functions of the volume fraction of the particles for various cases. The solutions for a parallel problem concerning the sedimentation of a suspension of identical spherical particles are also presented in the Appendix.

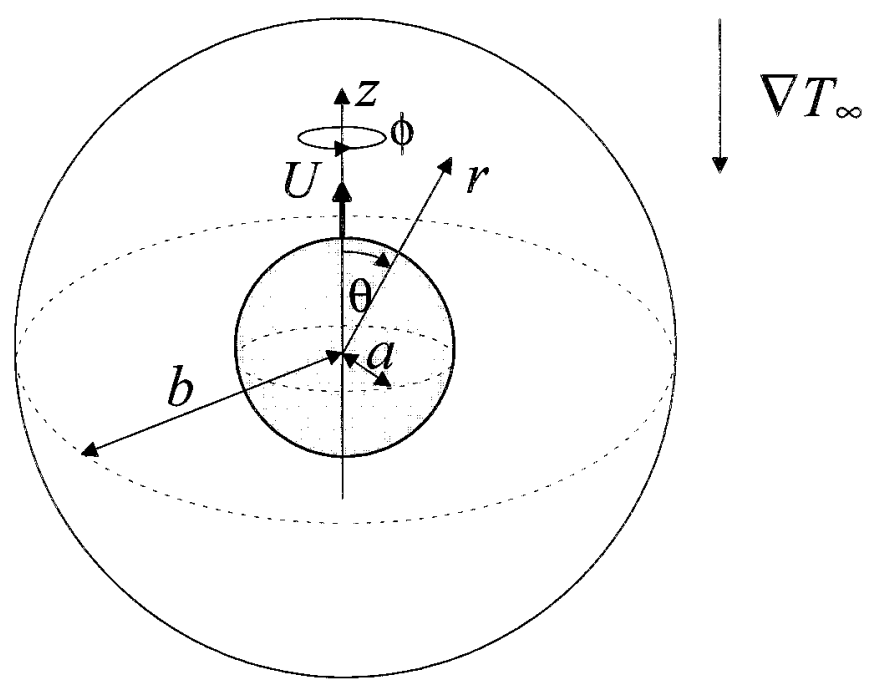

FIG. 1. Geometrical sketch for the thermophoretic motion of a spherical particle at the center of a spherical cell.

\section{ANALYSIS}

We consider the steady thermophoretic motion of a uniform three-dimensional distribution of identical spherical particles of arbitrary thermal conductivity and surface properties in a gaseous medium. The uniformly imposed temperature gradient $\nabla T_{\infty}$ equals $-E_{\infty} \mathbf{e}_{z}$ and the thermophoretic velocity of the particles is $U \mathbf{e}_{z}$, where $\mathbf{e}_{z}$ is the unit vector in the positive $z$ direction. As shown in Fig. 1, we employ a unit cell model (19, 20) in which each particle of radius $a$ is surrounded by a concentric spherical shell of suspending fluid having an outer radius of $b$ such that the particle/cell volume ratio is equal to the particle volume fraction $\varphi$ throughout the entire suspension; viz., $\varphi=(a / b)^{3}$. The Knudsen numbers $l / a$ and $l /(b-$ a) are assumed to be small so that the fluid flow is in the continuum regime and the Knudsen layers surrounding the particles do not overlap with one another. (If the shell volume of order $b^{3}-a^{3}$ is sufficiently small, then the characteristics of the gas in this volume are subject to statistical fluctuation.) The origin of the spherical coordinate system $(r, \theta, \phi)$ is set at the center of the particle. Our objective is to determine the particle velocity $U$ in a cell induced by thermophoretic force.

The Peclet number of this axisymmetric problem is assumed to be small. Hence, the equation of energy governing the temperature distribution $T(r, \theta)$ for the fluid of constant thermal conductivity $k$ is Laplace's equation:

$$
\nabla^{2} T=0 \quad(a \leq r \leq b) .
$$

For the temperature field $T_{1}(r, \theta)$ inside the particle, one has

$$
\nabla^{2} T_{1}=0 \quad(r \leq a)
$$


The boundary conditions at the particle surface require that the normal component of heat flux be continuous and a temperature jump which is proportional to the normal temperature gradient (4) occur. Also, the local temperature gradient at the outer (virtual) surface of the cell is parallel to the uniformly applied gradient $-E_{\infty} \mathbf{e}_{z}$. Thus,

$$
\begin{gathered}
r=a: \quad k \frac{\partial T}{\partial r}=k_{1} \frac{\partial T_{1}}{\partial r}, \\
T-T_{1}=C_{\mathrm{t}} l \frac{\partial T}{\partial r} \\
r=b: \quad \frac{\partial T}{\partial r}=-E_{\infty} \cos \theta,
\end{gathered}
$$

where $k_{1}$ is the thermal conductivity of the particle and $C_{\mathrm{t}}$ is the temperature jump coefficient about the surface of the particle, both are assumed to be constant.

The solution to Eqs. [2]-[4] is

$$
\begin{gathered}
T_{1}=T_{0}-3 A E_{\infty} r \cos \theta \quad(r \leq a), \\
T=T_{0}-A\left[2+k^{*}+2 k^{*} C_{\mathrm{t}}^{*}+\left(1-k^{*}+k^{*} C_{\mathrm{t}}^{*}\right) \frac{a^{3}}{r^{3}}\right] \\
\times E_{\infty} r \cos \theta \quad(a \leq r \leq b),
\end{gathered}
$$

where

$$
A=\left[2+k^{*}+2 k^{*} C_{\mathrm{t}}^{*}-2\left(1-k^{*}+k^{*} C_{\mathrm{t}}^{*}\right) \varphi\right]^{-1},
$$

$k^{*}=k_{1} / k, C_{\mathrm{t}}^{*}=C_{\mathrm{t}} l / a, \varphi=(a / b)^{3}$, and $T_{0}$ is the temperature at the center of the particle. It is understood that the practical value of $C_{\mathrm{t}}^{*}$ is less than unity with the assumption of a small Knudsen number. Note that the dimensionless parameter $k^{*} C_{\mathrm{t}}^{*}$ denotes the relative resistance caused by the temperature jump at the particle surface with respect to the heat conduction inside the particle.

The boundary condition of the temperature at the virtual surface $r=b$ may be taken as the distribution giving rise to the gradient $-E_{\infty} \mathbf{e}_{z}$ in the cell when the particle does not exist. In this case, Eq. [4] becomes

$$
r=b: \quad T=T_{0}-E_{\infty} r \cos \theta .
$$

The solution to the governing Eqs. [2a] and [2b] subject to the boundary conditions [3] and [7] is also given by the form of Eq. [5], but with parameter $A$ defined as

$$
A=\left[2+k^{*}+2 k^{*} C_{\mathrm{t}}^{*}+\left(1-k^{*}+k^{*} C_{\mathrm{t}}^{*}\right) \varphi\right]^{-1} .
$$

For the special case of $k^{*}=\left(1-C_{\mathrm{t}}^{*}\right)^{-1}$, Eqs. [6] and [8] both give $A=1 / 3 k^{*}$, and the temperature gradient in the fluid phase resulting from Eq. [5b] equals the constant imposed value everywhere.

With knowledge of the solution for the temperature distribution, we can now proceed to find the flow field in a cell. The fluid surrounding the particle is assumed to be incompressible and Newtonian. Due to the low Reynolds number, the fluid motion caused by the thermophoretic motion of the particle is governed by the quasi-steady fourth-order differential equation for viscous axisymmetric flows,

$$
E^{4} \Psi=E^{2}\left(E^{2} \Psi\right)=0 \quad(a \leq r \leq b),
$$

where $\Psi(r, \theta)$ is the Stokes stream function. In spherical coordinates, the Stokesian operator $E^{2}$ is given by

$$
E^{2}=\frac{\partial^{2}}{\partial r^{2}}+\frac{\sin \theta}{r^{2}} \frac{\partial}{\partial \theta}\left(\frac{1}{\sin \theta} \frac{\partial}{\partial \theta}\right) \text {. }
$$

The stream function $\Psi$ is related to the $r$ and $\theta$ components of the fluid velocity field $\mathbf{v}$ by

$$
\begin{aligned}
& \mathrm{v}_{\mathrm{r}}=-\frac{1}{r^{2} \sin \theta} \frac{\partial \Psi}{\partial \theta} \\
& \mathrm{v}_{\theta}=\frac{1}{r \sin \theta} \frac{\partial \Psi}{\partial r} .
\end{aligned}
$$

Owing to the thermal creep and frictional slip velocities along the solid-fluid interface, the boundary conditions for the fluid velocity at the particle surface are (12)

$$
\begin{aligned}
r=a: \quad \mathrm{V}_{\mathrm{r}} & =U \cos \theta, \\
\mathrm{V}_{\theta} & =-U \sin \theta+\frac{C_{\mathrm{m}} l}{\eta} \tau_{\mathrm{r} \theta}+\frac{C_{\mathrm{s}} \eta}{\rho T_{0}} \frac{\partial T}{r \partial \theta} .
\end{aligned}
$$

Here, $C_{\mathrm{m}}$ and $C_{\mathrm{s}}$ are the frictional slip and thermal creep coefficients, respectively, about the surface of the particle; $\tau_{\mathrm{r} \theta}$ is the shear stress for the fluid flow,

$$
\tau_{\mathrm{r} \theta}=\eta\left[r \frac{\partial}{\partial r}\left(\frac{\mathrm{V}_{\theta}}{r}\right)+\frac{1}{r} \frac{\partial \mathrm{V}_{\mathrm{r}}}{\partial \theta}\right],
$$

and $U$ is the thermophoretic velocity of the particle to be determined. The derivative $\partial T / \partial \theta$ can be evaluated from the temperature distribution given by Eq. [5]. The validity of the expression for the thermal creep velocity in Eq. [12b] is based on the assumption that the fluid is only slightly nonuniform in the undisturbed temperature on the length scale of the particle radius. On the outer (virtual) boundary of the cell, the Happel 
(19) model assumes that the radial velocity and the shear stress are zero; viz.,

$$
\begin{aligned}
r=b: \quad \mathrm{v}_{\mathrm{r}} & =0, \\
\tau_{\mathrm{r} \theta} & =0 .
\end{aligned}
$$

A solution to Eq. [9] suitable for satisfying boundary conditions on the spherical surfaces is (22)

$$
\Psi=\left(C r^{-1}+D r+E r^{2}+F r^{4}\right) \sin ^{2} \theta .
$$

The constants $C, D, E$, and $F$ are to be determined from Eqs. [12] and [14] using Eq. [11]. The procedure is straightforward, with the result

$$
\begin{aligned}
C= & a^{3} \omega\left[U-3 A\left(1-\varphi^{1 / 3}\right) V\right], \\
D= & -a \omega\left\{\left[3\left(1+2 C_{\mathrm{m}}^{*}\right)+2\left(1-3 C_{\mathrm{m}}^{*}\right) \varphi^{5 / 3}\right] U\right. \\
& \left.-3 A\left(1-\varphi^{5 / 3}\right) V\right\}, \\
E= & \omega\left\{\left[3\left(1+2 C_{\mathrm{m}}^{*}\right) \varphi^{1 / 3}+2\left(1-3 C_{\mathrm{m}}^{*}\right) \varphi^{2}\right] U\right. \\
& \left.-3 A\left(\varphi^{1 / 3}-\varphi^{2}\right) V\right\}, \\
F= & -a^{-2} \omega\left[\varphi^{5 / 3} U-3 A\left(\varphi^{5 / 3}-\varphi^{2}\right) V\right],
\end{aligned}
$$

where $C_{\mathrm{m}}^{*}=C_{\mathrm{m}} l / a$,

$$
V=\frac{2 C_{\mathrm{s}} \eta}{\rho T_{0}}\left(1+k^{*} C_{\mathrm{t}}^{*}\right) E_{\infty}
$$

which is a characteristic migration velocity of the particle, and

$$
\begin{aligned}
\omega=\left[2 \left(2-3 \varphi^{1 / 3}\right.\right. & \left.+3 \varphi^{5 / 3}-2 \varphi^{2}\right) \\
& \left.+12 C_{\mathrm{m}}^{*}\left(1-\varphi^{1 / 3}-\varphi^{5 / 3}+\varphi^{2}\right)\right]^{-1} .
\end{aligned}
$$

With this solution, the components of the fluid velocity in this axisymmetric flow (with $\mathrm{v}_{\phi}=0$ ) can be calculated by using Eq. [11].

The drag force (in the $z$ direction) exerted by the fluid on the particle is (22)

$$
F_{\mathrm{d}}=8 \pi \eta D
$$

Because the particle is freely suspended in the fluid, the net force exerted by the fluid on the particle must vanish; viz., $D=$ 0. With this constraint, Eq. [16b] yields the thermophoretic velocity of the particle,

$$
U=3 A\left(1-\varphi^{5 / 3}\right)\left[3\left(1+2 C_{\mathrm{m}}^{*}\right)+2\left(1-3 C_{\mathrm{m}}^{*}\right) \varphi^{5 / 3}\right]^{-1} V
$$

where $A$ is given by Eqs. [6] or [8].

If the Kuwabara (20) model for the boundary conditions of the fluid flow at the virtual surface of the cell, which assumes that the radial velocity and the vorticity are zero, is used, Eq. [14b] is replaced by

$$
r=b: \quad(\nabla \times \mathbf{v})_{\phi}=\frac{\partial \mathrm{V}_{\theta}}{\partial r}+\frac{\mathrm{V}_{\theta}}{r}-\frac{1}{r} \frac{\partial \mathrm{V}_{\mathrm{r}}}{\partial \theta}=0 .
$$

With this change, the stream function $\Psi$ can still be expressed in the form of Eq. [15], but the coefficients $C, D, E$, and $F$ are replaced by $C^{\prime}, D^{\prime}, E^{\prime}$, and $F^{\prime}$, respectively, which should be determined by boundary conditions [12], [14a], and [21]. The result is

$$
\begin{aligned}
C^{\prime}= & a^{3} \omega^{\prime}\left[\left(5-2 \varphi+6 C_{\mathrm{m}}^{*} \varphi\right) U\right. \\
& \left.-3 A\left(5-6 \varphi^{1 / 3}+\varphi\right) V\right], \\
D^{\prime}= & -15 a \omega^{\prime}\left[\left(1+2 C_{\mathrm{m}}^{*}\right) U-A(1-\varphi) V\right], \\
E^{\prime}=\omega^{\prime}\left\{\left[18 \varphi^{1 / 3}-5 \varphi+2 \varphi^{2}+6 C_{\mathrm{m}}^{*}\left(6 \varphi^{1 / 3}-\varphi^{2}\right)\right] U\right. & \left.-3 A\left(6 \varphi^{1 / 3}-5 \varphi-\varphi^{2}\right) V\right\}, \\
F^{\prime}= & -3 a^{-2} \omega^{\prime}\left[\left(1+2 C_{\mathrm{m}}^{*}\right) \varphi U-A\left(\varphi-\varphi^{2}\right) V\right],
\end{aligned}
$$

where

$$
\begin{aligned}
\omega^{\prime}=\left[4 \left(5-9 \varphi^{1 / 3}+\right.\right. & \left.5 \varphi-\varphi^{2}\right) \\
& \left.+12 C_{\mathrm{m}}^{*}\left(5-6 \varphi^{1 / 3}+\varphi^{2}\right)\right]^{-1} .
\end{aligned}
$$

The fact that there is no drag force exerted on the particle requires $D^{\prime}=0$, and Eq. [22b] gives the particle velocity as

$$
U=A(1-\varphi)\left(1+2 C_{\mathrm{m}}^{*}\right)^{-1} V
$$

For the sedimentation of a suspension of identical spherical particles considered in the Appendix, both the Happel and the Kuwabara models give qualitatively the same flow fields and approximately comparable drag forces. However, the Happel model has a significant advantage in that it does not require an exchange of mechanical energy between the cell and the environment (22).

\section{RESULTS AND DISCUSSION}

Due to the differences in the boundary conditions for the temperature and velocity distributions at the virtual surface $r=$ $b$ of the unit cell, four cases of the cell model can be defined: 
Case I: the Happel model with a specified temperature gradient at the virtual surface (the boundary conditions at $r=$ $b$ are described by Eqs. [4] and [14]);

Case II: the Happel model with a specified temperature profile at the virtual surface (the boundary conditions at $r=b$ are described by Eqs. [7] and [14]);

Case III: the Kuwabara model with a specified temperature gradient at the virtual surface (the boundary conditions at $r=$ $b$ are described by Eqs. [4], [14a], and [21]);

Case IV: the Kuwabara model with a specified temperature profile at the virtual surface (the boundary conditions at $r=b$ are described by Eqs. [7], [14a], and [21]).

The analytical solutions of the temperature and flow fields in the unit cell and the thermophoretic velocity of the particle have been obtained in the previous section for all of the four cases.

In Case I, the particle velocity is given by Eq. [20] with coefficient $A$ defined by Eq. [6]. This migration velocity can be expressed as

$$
\begin{aligned}
U=U^{(0)}\left(1-\varphi^{5 / 3}\right)[1 & \left.-\frac{2\left(1-k^{*}+k^{*} C_{\mathrm{t}}^{*}\right)}{2+k^{*}+2 k^{*} C_{\mathrm{t}}^{*}} \varphi\right]^{-1} \\
& \times\left[1+\frac{2\left(1-3 C_{\mathrm{m}}^{*}\right)}{3\left(1+2 C_{\mathrm{m}}^{*}\right)} \varphi^{5 / 3}\right]^{-1}
\end{aligned}
$$

where

$$
U^{(0)}=\frac{2\left(1+k^{*} C_{\mathrm{t}}^{*}\right)}{\left(1+2 C_{\mathrm{m}}^{*}\right)\left(2+k^{*}+2 k^{*} C_{\mathrm{t}}^{*}\right)} \frac{C_{\mathrm{s}} \eta}{\rho T_{0}} E_{\infty},
$$

which is the thermophoretic velocity of the particle given by Eq. [1] in the limit $\varphi=0$. The particle velocity $U$ can also be written as the following power expansion in $\varphi$ :

$$
U=U^{(0)}\left[1+\gamma \varphi+\beta \varphi^{5 / 3}+O\left(\varphi^{2}\right)\right] .
$$

A comparison between Eqs. [25] and [27] leads to the coefficients $\gamma$ and $\beta$ for Case I,

$$
\begin{aligned}
& \gamma_{\mathrm{I}}=\frac{2-2 k^{*}+2 k^{*} C_{\mathrm{t}}^{*}}{2+k^{*}+2 k^{*} C_{\mathrm{t}}^{*}}, \\
& \beta_{\mathrm{I}}=-\frac{5}{3\left(1+2 C_{\mathrm{m}}^{*}\right)} .
\end{aligned}
$$

Note that $\gamma_{\mathrm{I}}$ is only related to the parameters $k^{*}$ and $C_{\mathrm{t}}^{*}$, and $\beta_{\mathrm{I}}$ is a function of the parameter $C_{\mathrm{m}}^{*}$ only. One can find $\gamma_{\mathrm{I}}=0$ if $k^{*}=\left(1-C_{\mathrm{t}}^{*}\right)^{-1}, 0<\gamma_{\mathrm{I}} \leq 1$ for $0 \leq k^{*}<\left(1-C_{\mathrm{t}}^{*}\right)^{-1}$, and $-2\left(1-C_{\mathrm{t}}^{*}\right)\left(1+2 C_{\mathrm{t}}^{*}\right)^{-1}<\gamma_{\mathrm{I}}<0$ for $k^{*}>\left(1-C_{\mathrm{t}}^{*}\right)^{-1}$ $\left(\gamma_{\mathrm{I}}\right.$ is a monotonically decreasing function of $\left.k^{*}\right)$. Also, $\beta_{\mathrm{I}}=$ $-\frac{5}{3}$ if $C_{\mathrm{m}}^{*}=0$ and $-\frac{5}{3}<\beta_{\mathrm{I}}<0$ for $C_{\mathrm{m}}^{*}>0\left(\beta_{\mathrm{I}}\right.$ is a monotonically increasing function of $C_{\mathrm{m}}^{*}$ ).
In Case II, the thermophoretic velocity of the particle can be evaluated by Eq. [20] with $A$ given by Eq. [8], and its expression parallel to Eq. [25] for Case I is

$$
\begin{aligned}
U=U^{(0)}\left(1-\varphi^{5 / 3}\right)(1 & \left.+\frac{1-k^{*}+k^{*} C_{\mathrm{t}}^{*}}{2+k^{*}+2 k^{*} C_{\mathrm{t}}^{*}} \varphi\right)^{-1} \\
& \times\left[1+\frac{2\left(1-3 C_{\mathrm{m}}^{*}\right)}{3\left(1+2 C_{\mathrm{m}}^{*}\right)} \varphi^{5 / 3}\right]^{-1} .
\end{aligned}
$$

This particle velocity can also be expressed in the expansion form of Eq. [27], with the coefficients $\gamma$ and $\beta$ given by

$$
\begin{aligned}
& \gamma_{\mathrm{II}}=-\frac{1-k^{*}+k^{*} C_{\mathrm{t}}^{*}}{2+k^{*}+2 k^{*} C_{\mathrm{t}}^{*},} \\
& \beta_{\mathrm{II}}=-\frac{5}{3\left(1+2 C_{\mathrm{m}}^{*}\right)} .
\end{aligned}
$$

Here, $\beta_{\mathrm{II}}$ is the same as $\beta_{\mathrm{I}}$ in Case I, and again, Eq. [30a] yields $\gamma_{\mathrm{II}}=0$ if $k^{*}=\left(1-C_{\mathrm{t}}^{*}\right)^{-1}$. However, in this case, $-\frac{1}{2} \leq \gamma_{\mathrm{II}}<$ 0 for $0 \leq k^{*}<\left(1-C_{\mathrm{t}}^{*}\right)^{-1}$ and $0<\gamma_{\mathrm{II}}<\left(1-\mathrm{C}_{\mathrm{t}}^{*}\right)(1+$ $\left.2 C_{\mathrm{t}}^{*}\right)^{-1}$ for $k^{*}>\left(1-C_{\mathrm{t}}^{*}\right)^{-1}\left(\gamma_{\mathrm{II}}\right.$ is a monotonically increasing function of $\left.k^{*}\right)$, which is opposite to the corresponding tendency in Case I ( $\gamma_{\mathrm{II}}$ in this case equals the value of $\gamma_{\mathrm{I}}$ in Case I multiplied by $\left.-\frac{1}{2}\right)$.

In Case III, the thermophoretic velocity of the particle can be obtained by the substitution of Eq. [6] into Eq. [24], with the result

$$
U=U^{(0)}(1-\varphi)\left[1-\frac{2\left(1-k^{*}+k^{*} C_{\mathrm{t}}^{*}\right)}{2+k^{*}+2 k^{*} C_{\mathrm{t}}^{*}} \varphi\right]^{-1}
$$

When this velocity is expressed in the expansion form of Eq. [27], one has

$$
\begin{aligned}
& \gamma_{\mathrm{III}}=-\frac{3 k^{*}}{2+k^{*}+2 k^{*} C_{\mathrm{t}}^{*}}, \\
& \beta_{\mathrm{III}}=0 .
\end{aligned}
$$

Equation [32a] indicates $\gamma_{\mathrm{III}}=0$ if $k^{*}=0, \gamma_{\mathrm{III}}=-1$ if $k^{*}=$ $\left(1-C_{\mathrm{t}}^{*}\right)^{-1}$, and $-3\left(1+2 C_{\mathrm{t}}^{*}\right)^{-1}<\gamma_{\mathrm{III}}<0$ for $k^{*}>0\left(\gamma_{\mathrm{III}}\right.$ in this case equals the value of $\gamma_{\mathrm{I}}$ in Case I minus 1).

In Case IV, the particle velocity is given by Eq. [24] with $A$ defined by Eq. [8], and it can be expressed as

$$
U=U^{(0)}(1-\varphi)\left(1+\frac{1-k^{*}+k^{*} C_{\mathrm{t}}^{*}}{2+k^{*}+2 k^{*} C_{\mathrm{t}}^{*}} \varphi\right)^{-1}
$$

When this formula is written in the form of Eq. [27], one has 


$$
\begin{aligned}
& \gamma_{\mathrm{IV}}=-\frac{3+3 k^{*} C_{\mathrm{t}}^{*}}{2+k^{*}+2 k^{*} C_{\mathrm{t}}^{*}}, \\
& \beta_{\mathrm{IV}}=0 .
\end{aligned}
$$

As in Case III, $\gamma_{\mathrm{IV}}=-1$ if $k^{*}=\left(1-C_{\mathrm{t}}^{*}\right)^{-1}$ and $\beta_{\mathrm{IV}}=0$. However, here $\gamma_{\mathrm{IV}}=-\frac{3}{2}$ if $k^{*}=0$ and $-\frac{3}{2}<\gamma_{\mathrm{IV}}<$ $-3 C_{\mathrm{t}}^{*}\left(1+2 C_{\mathrm{t}}^{*}\right)^{-1}$ for $k^{*}>0\left(\gamma_{\mathrm{IV}}\right.$ in this case equals the value of $\gamma_{\text {II }}$ in Case II minus 1).

It can be found from Eqs. [25]-[34] that the mean thermophoretic velocity in a homogeneous suspension of identical spherical particles predicted by the cell model is quite sensitive to the boundary conditions specified at the virtual surface of the cell. The boundary condition for the temperature distribution at the virtual surface $r=b$ determines the dependence of the normalized particle velocity (or mobility) $U / U^{(0)}$ as a function of $k^{*}$ and $C_{\mathrm{t}}^{*}$, while the boundary condition for the velocity field at $r=b$ controls the connection of $U / U^{(0)}$ with $C_{\mathrm{m}}^{*}$. Also, the Kuwabara cell model (described by Case III and Case IV) predicts that $U / U^{(0)}$ is independent of the value of $C_{\mathrm{m}}^{*}$. For given values of $k^{*}, C_{\mathrm{t}}^{*}, C_{\mathrm{m}}^{*}$, and $\varphi$, the mean particle velocities obtained from Case III and Case IV are always smaller than those obtained from Case I and Case II, respectively.

The effect of particle concentration on thermophoresis is much weaker than that on sedimentation, which is analyzed by using the unit cell model in the Appendix. As seen in Eq. [27], the leading order of this effect is $\varphi$ for thermophoretic motion, compared with $\varphi^{1 / 3}$ in Eqs. [A4] and [A6] for sedimentation. The reason for the weaker concentration effect on thermophoresis is that the disturbance to the fluid velocity field caused by a thermophoretic particle decays faster (as $r^{-3}$ ) than that caused by an aerosol particle moving under the influence of a body force (as $r^{-1}$ ).

Based on the analytical (approximate) solutions of the thermal and hydrodynamic interactions between pairs of aerosol spheres in a uniformly prescribed temperature gradient obtained by a method of reflections correct to $O\left(r_{12}^{-7}\right)$, where $r_{12}$ is the center-to-center distance between the particles, Keh and Chen (15) also derived a formula of the mean thermophoretic velocity in a dilute gas-particle system (say, $\varphi<0.1$ ) in the expansion form of Eq. [27] with $\beta=0$ by using the concepts of statistical mechanics. This ensemble-averaged result gives the coefficient $\gamma$ approximately as

$$
\gamma=-1-G+\frac{1}{8}\left[2 G^{2}-(1+6 H) G-\frac{5\left(1+2 C_{\mathrm{m}}^{*}\right)}{2\left(1+5 C_{\mathrm{m}}^{*}\right)}\right],
$$

where

$$
G=\frac{1-k^{*}+k^{*} C_{\mathrm{t}}^{*}}{2+k^{*}+2 k^{*} C_{\mathrm{t}}^{*}}
$$

$$
H=-\frac{5}{4} \frac{\left(1+2 k^{*} C_{\mathrm{t}}^{*}\right)\left(2+k^{*}+2 k^{*} C_{\mathrm{t}}^{*}\right)\left(1+2 C_{\mathrm{m}}^{*}\right)}{\left(1+k^{*} C_{\mathrm{t}}^{*}\right)\left(3+2 k^{*}+6 k^{*} C_{\mathrm{t}}^{*}\right)\left(1+5 C_{\mathrm{m}}^{*}\right)}
$$

In this statistical model, $\beta$ vanishes, which is similar to that in the Kuwabara cell model. Note that $\gamma$ is a function of all parameters $k^{*}, C_{\mathrm{t}}^{*}$, and $C_{\mathrm{m}}^{*}$ in Eq. [35], which is a sharp contrast to the prediction from the cell models that $\gamma$ is dependent on $k^{*}$ and $C_{\mathrm{t}}^{*}$ only. Equation [35] gives that, in general cases, the value of $\gamma$ (which is always negative) increases with the increase of $k^{*}$ for constant values of $C_{\mathrm{t}}^{*}$ and $C_{\mathrm{m}}^{*}$. As examples of limiting situations, Eq. [35] yields $\gamma=-\frac{3}{2}$ if $k^{*}=$ $0, \gamma=-\frac{7}{8}$ for the case of large particles $(l / a \ll 1)$ with $k^{*} \rightarrow$ $\infty$, and $\gamma=-13 / 32$ for the case of $l / a=0$ and $k^{*} \gg 1$.

On the other hand, Keh and Chen (18) used a boundary collocation method to obtain accurate information on the thermal and hydrodynamic interactions of two arbitrary aerosol spheres at all separations between the particles. Based on this information they also calculated the ensemble-averaged values of the coefficient $\gamma$ for various values of $k^{*}$ for the representative case of $C_{\mathrm{t}}^{*}=2 C_{\mathrm{m}}^{*}=0.2$. It was found that the value of $\gamma$ increases monotonically with the increase of $k^{*}$ for given values of $C_{\mathrm{t}}^{*}$ and $C_{\mathrm{m}}^{*}$. Also, the results of $\gamma$ calculated from the approximate formula [35] always overestimate its exact values and the error can be quite significant.

A sketch of the heat conduction lines (with a constant heat conduction function $Q$ for each) and the isotherms around a spherical particle situated at the center of a spherical cell with $a / b=0.5$ is exhibited in Fig. 2 for the temperature field prescribed by either Eq. [4] or Eq. [7]. In this axisymmetric case, the heat conduction function is orthogonally related to the temperature distribution by the formulas (23)

$$
\begin{aligned}
& \frac{\partial Q}{\partial R}=R \frac{\partial T}{\partial z}, \\
& \frac{\partial Q}{\partial z}=-R \frac{\partial T}{\partial R},
\end{aligned}
$$

where $R=r \sin \theta$, which is the radial cylindrical coordinate. These relations can be integrated after the substitution of the temperature profile provided by Eq. [5] to yield

$$
\begin{gathered}
Q_{1}=-\frac{3}{2} A E_{\infty} r^{2} \sin ^{2} \theta \quad(r \leq a), \\
Q=-A\left[\frac{1}{2}\left(2+k^{*}+2 k^{*} C_{\mathrm{t}}^{*}\right)-\left(1-k^{*}+k^{*} C_{\mathrm{t}}^{*}\right) \frac{a^{3}}{r^{3}}\right] \\
\times E_{\infty} r^{2} \sin ^{2} \theta \quad(a \leq r \leq b),
\end{gathered}
$$

where the value of $Q$ along the $z$ axis (with $\theta=0$ and $\pi$ ) is set equal to zero for convenience. In Fig. 2, only the contours in 
half of a meridian plane (with $0 \leq \theta \leq \pi / 2$ ) are depicted due to the axial and fore/aft symmetry. It can be found that the heat conduction lines are attracted by the particle if $k^{*}\left(1-C_{\mathrm{t}}^{*}\right)>$ 1 , and they are repelled by the particle if $k^{*}\left(1-C_{\mathrm{t}}^{*}\right)<1$. Note that the isotherms are not continuous at the particle surface, as specified by Eq. [3a].

The streamline pattern for an isolated thermophoretic sphere in an unbounded fluid is available in the literature (24). The fluid streamlines (with a constant stream function $\Psi$ for each) around the particle undergoing thermophoresis in a cell are drawn in Fig. 3 for the case of $a / b=0.5$. The value of $k^{*}$ equal to $\left(1-C_{\mathrm{t}}^{*}\right)^{-1}$ is chosen so that the two cases of the Happel model (Case I and Case II) are the same, so are the two cases of the Kuwabara model (Case III and Case IV). As expected, a stagnation point is observed at $\theta=0$ and $z=b$ (and also at $\theta=\pi$ and $z=-b$ ). The fluid at $\theta=\pi / 2$ moves in the direction of the prescribed temperature gradient and, therefore, against the direction of particle movement. There is no qualitative difference in streamline pattern between the Happel and the Kuwabara cell models; however, the spacing between streamlines is narrower and the fluid recirculation is stronger for the Happel model, which shows a larger particle velocity. Note that (see Eqs. [31] and [33]) the streamlines displayed in Fig. $3 b$ for the Kuwabara model are independent of the value of $C_{\mathrm{m}}^{*}$.

The normalized thermophoretic velocity in a homogeneous suspension of identical spherical particles, $U / U^{(0)}$, as calculated from Eqs. [25], [29], [31], and [33] for the four cases of the cell model, is plotted versus the volume fraction of the particles, $\varphi$, in Figs. $4-8$ for various values of $k^{*}, C_{\mathrm{t}}^{*}$, and $C_{\mathrm{m}}^{*}$. In all cases, $U / U^{(0)}=1$ in the limit $\varphi=0$. The calculations are presented up to $\varphi=0.74$, which corresponds to the maximum attainable volume fraction for a swarm of identical spheres (25). It is also clear that at volume fractions approaching this, agglomeration due to contacts between particles may occur, and the present study does not cover this case. Figure 4 shows the results of $U / U^{(0)}$ as a function of $\varphi$ for a suspension of particles with $k^{*}=100$ and $C_{\mathrm{t}}^{*}=2 C_{\mathrm{m}}^{*}=0.2$. The results obtained from the statistical model (Eq. [27] with $\gamma=-0.430$ and $\beta=0$ (18)) for dilute gas-particle systems are also exhibited in this figure to compare with those of the cell model. In general, the thermophoretic velocity predicted by Case II is greater than that predicted by the statistical model and can be greater than that in the limit $\varphi=0$ in practical ranges, while the normalized velocity $U / U^{(0)}$ predicted by each of the other three cases of the cell model is a monotonically decreasing function of $\varphi$ and is smaller than that predicted by the statistical model. Note that the result for Case IV is quite close to that for the statistical model in dilute suspensions $(\varphi \rightarrow 0)$ of particles.

The results of $U / U^{(0)}$ as a function of $\varphi$ for a monodisperse suspension of particles with a small value of $k^{*}\left(k^{*}=0.1\right)$ and $C_{\mathrm{t}}^{*}=2 C_{\mathrm{m}}^{*}=0.2$ are plotted in Fig. 5 for both of the cases of the statistical model (Eq. [27] with $\gamma=-1.187$ and $\beta=0$ (18)) and the cell model. In general, the thermophoretic veloc-
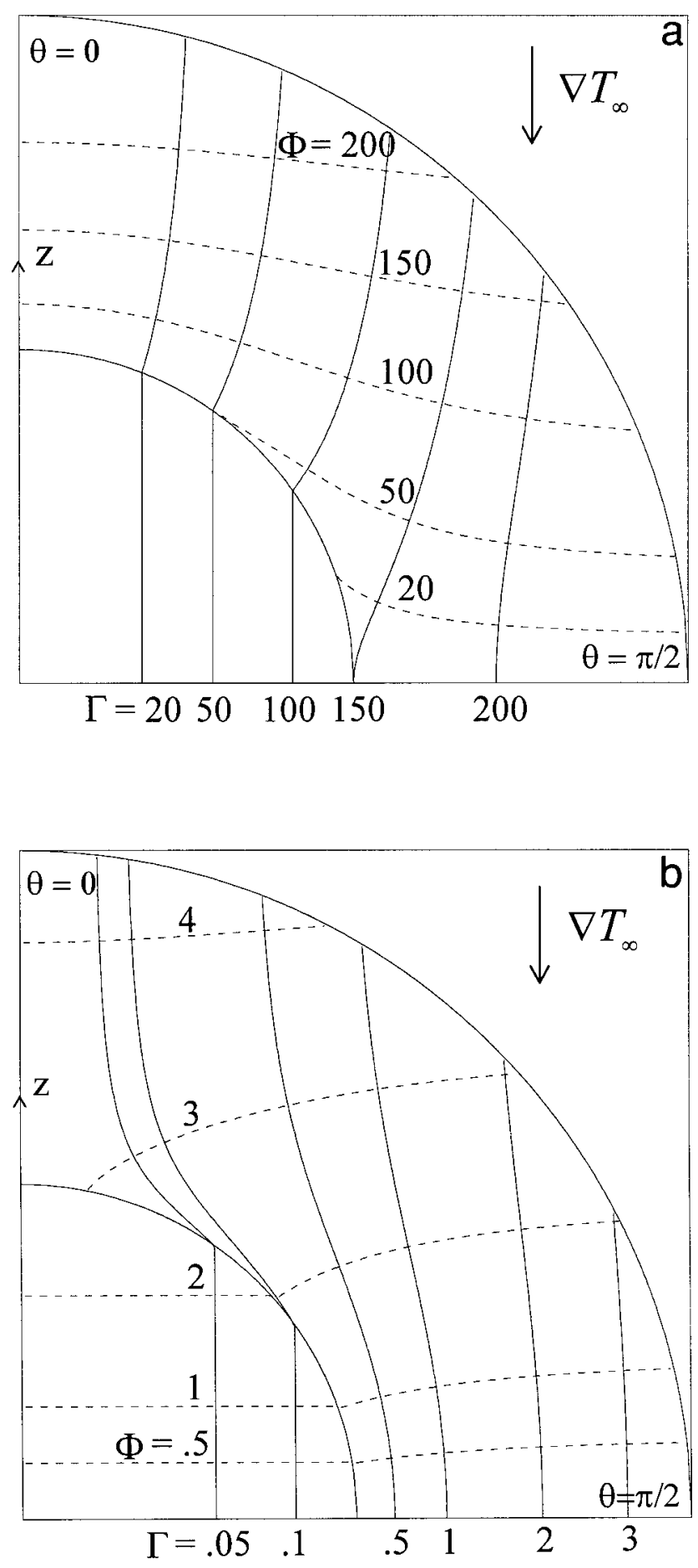

FIG. 2. Heat conduction lines (solid curves) and isotherms (dashed curves) around a spherical particle at the center of a spherical cell with $a / b=$ 0.5 and $C_{\mathrm{t}}^{*}=0.2$ : (a) $k^{*}=100$; (b) $k^{*}=0.1$. The dimensionless conduction function $\Gamma=-Q / A E_{\infty} a^{2}$ and the dimensionless temperature $\Phi=-(T-$ $\left.T_{0}\right) / A E_{\infty} a$.

ity predicted by Case IV is smaller than that predicted by the statistical model, while the normalized velocity $U / U^{(0)}$ predicted by each of the other three cases of the cell model is 

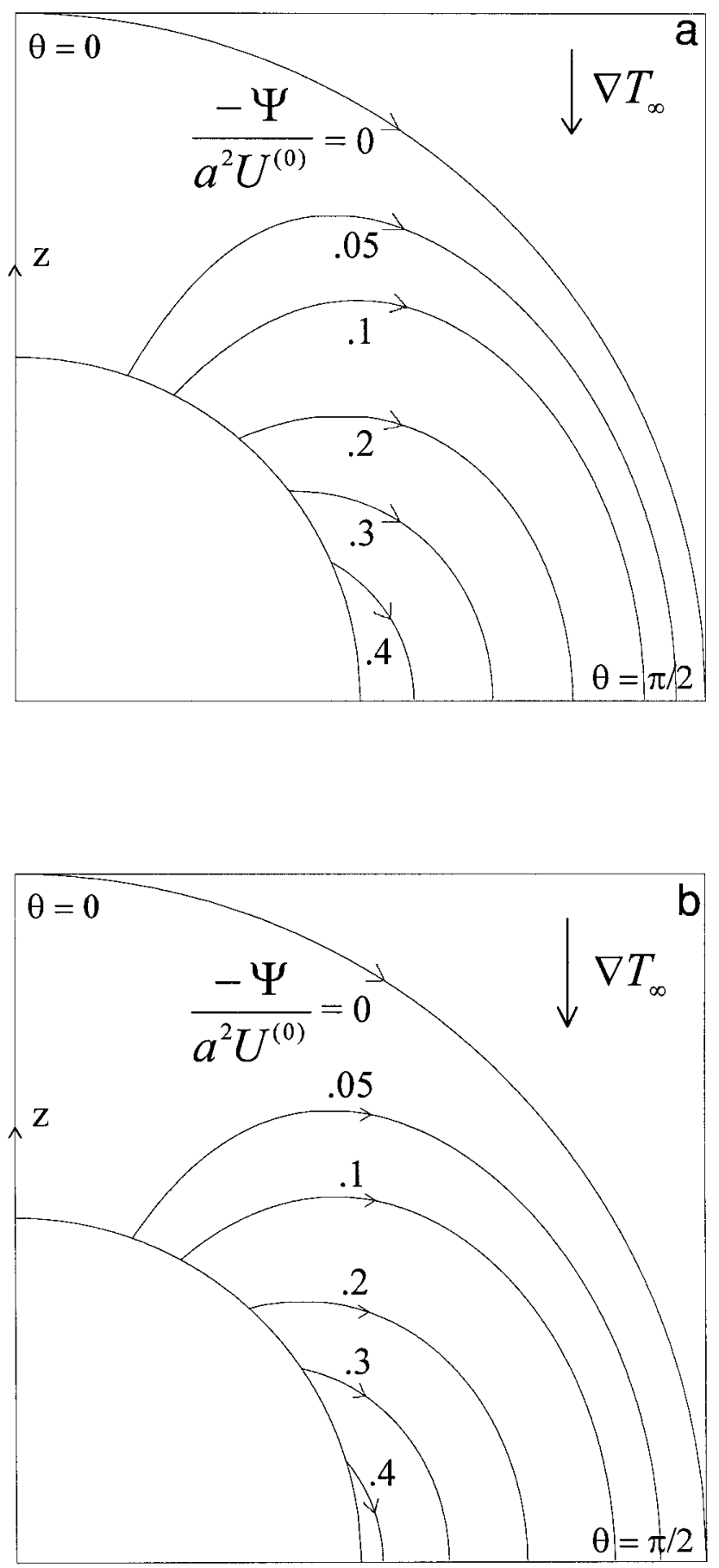

FIG . 3. Fluid streamlines around a spherical particle undergoing thermophoretic motion at the center of a spherical cell with $a / b=0.5$ and $k^{*}=(1-$ $\left.C_{\mathrm{t}}^{*}\right)^{-1}$ : (a) the Happel model with $C_{\mathrm{m}}^{*}=0.1$; (b) the Kuwabara model.

greater than that predicted by the statistical model. The value of $U / U^{(0)}$ obtained by Case I can be greater than that in the limit $\varphi=0$ in practical ranges. Again, similar to the situation of a suspension of particles with $k^{*}=100$, the result for Case IV is close to that for the statistical model in dilute systems.

In Fig. 6, the normalized thermophoretic mobility $U / U^{(0)}$ for a cloud of identical particles uniformly suspended in a gas medium with $k^{*}=\left(1-C_{\mathrm{t}}^{*}\right)^{-1}$ is plotted as a function of $\varphi$ for the Happel cell model (Case I and Case II, which are the same with $\left.k^{*}=\left(1-C_{\mathrm{t}}^{*}\right)^{-1}\right)$ with $C_{\mathrm{m}}^{*}$ as a parameter. It can be seen in this figure that $U / U^{(0)}$ is independent of $\varphi$ in the special situation of $C_{\mathrm{m}}^{*} \rightarrow \infty$, but decreases monotonically with the increase of $\varphi$ for any finite value of $C_{\mathrm{m}}^{*}$. Also, $U / U^{(0)}$ is a monotonically increasing function of $C_{\mathrm{m}}^{*}$ for a given value of $\varphi$, similar to their relation in the case of sedimentation displayed by Fig. A2. Examining Eqs. [25] and [29], one can find that this tendency between $U / U^{(0)}$ and $C_{\mathrm{m}}^{*}$ also exists for all values of $k^{*}$ other than $\left(1-C_{\mathrm{t}}^{*}\right)^{-1}$.

Figure 7 shows plots of the normalized velocity $U / U^{(0)}$ for a suspension of identical particles with $C_{\mathrm{t}}^{*}=2 C_{\mathrm{m}}^{*}=0.2$ as a function of $\varphi$ for the Case I and Case II of the cell model with $k^{*}$ as a parameter. For a fixed value of $\varphi, U / U^{(0)}$ decreases monotonically with the increase of $k^{*}$ in Case I (opposite to the ensemble-averaged results), but increases monotonically with the increase of $k^{*}$ in Case II. For Case I with $k^{*}<\left(1-C_{\mathrm{t}}^{*}\right)^{-1}$ $(=1.25)$ or Case II with $k^{*}>\left(1-C_{\mathrm{t}}^{*}\right)^{-1}, U / U^{(0)}$ increases with the increase of $\varphi$ in a dilute gas-particle system, while this normalized velocity is a monotonically decreasing function of $\varphi$ for Case I with $k^{*} \geq\left(1-C_{\mathrm{t}}^{*}\right)^{-1}$ or Case II with $k^{*} \leq(1-$ $\left.C_{\mathrm{t}}^{*}\right)^{-1}$. Examining Eqs. [25] and [29], one can find that this trend of dependence of $U / U^{(0)}$ on $k^{*}$ and $\varphi$ also exists for any values of $C_{\mathrm{t}}^{*}$ and $C_{\mathrm{m}}^{*}$ other than 0.2 and 0.1 , respectively.

Figure 8 shows plots of the normalized velocity $U / U^{(0)}$ for a suspension of identical particles with $C_{\mathrm{t}}^{*}=0.2$ as a function

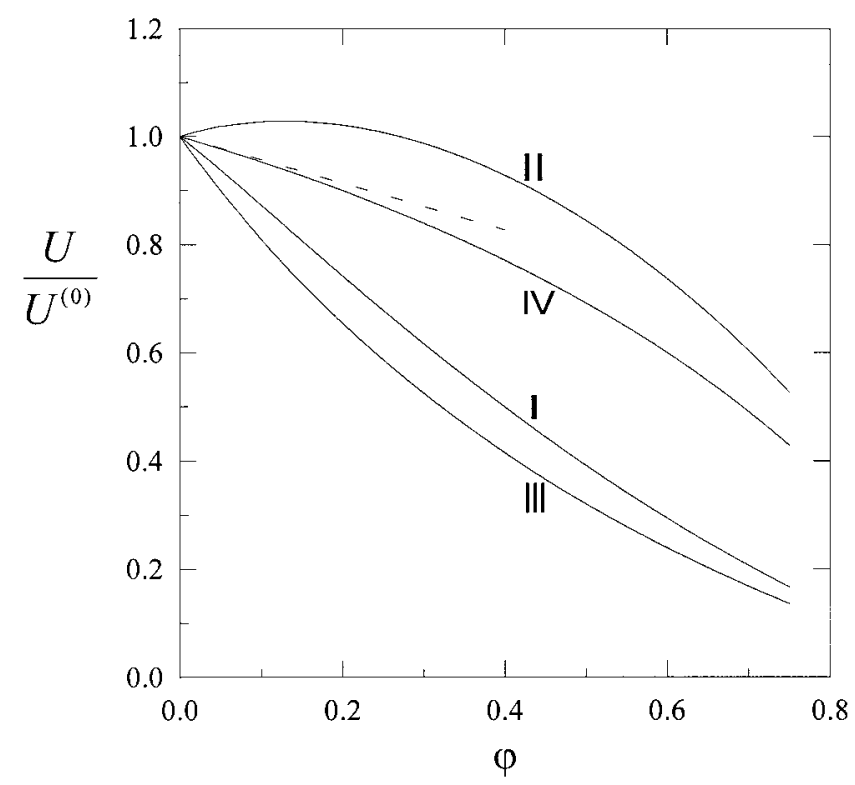

FIG . 4. Plots of the normalized thermophoretic velocity in a monodisperse suspension of spherical particles with $k^{*}=100$ and $C_{\mathrm{t}}^{*}=2 C_{\mathrm{m}}^{*}=0.2$ versus the volume fraction of the particles. The solid curves with labels I, II, III, and IV represent the cell-model calculations from Eqs. [25], [29], [31], and [33], respectively, and the dashed curve is the statistical-model result calculated from Eq. [27]. 


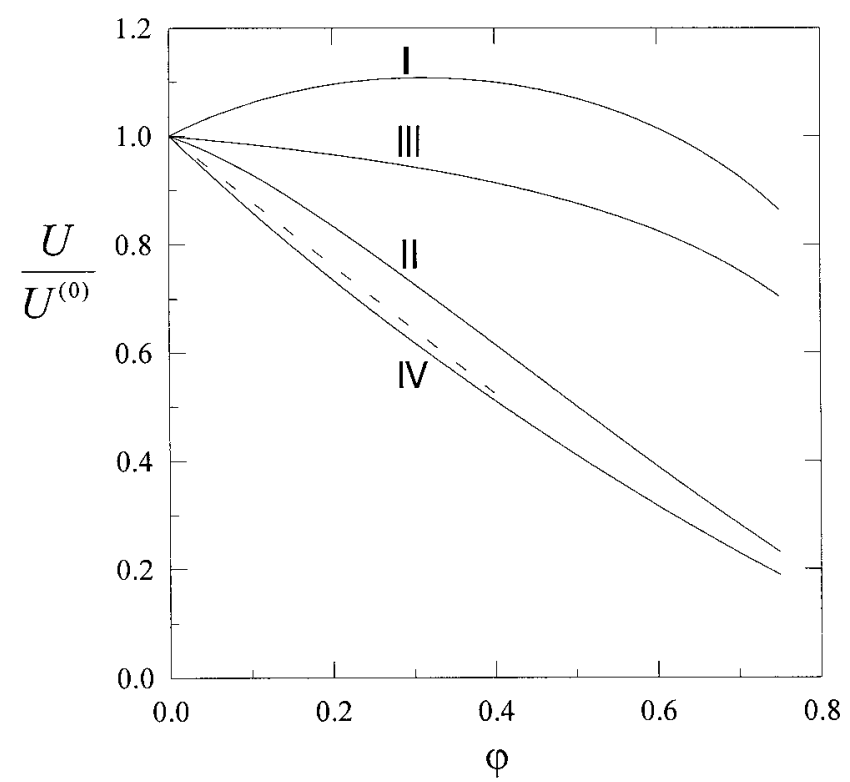

FIG . 5. Plots of the normalized thermophoretic velocity in a monodisperse suspension of spherical particles with $k^{*}=0.1$ and $C_{\mathrm{t}}^{*}=2 C_{\mathrm{m}}^{*}=0.2$ versus the volume fraction of the particles. The solid curves with labels I, II, III, and IV represent the cell-model calculations from Eqs. [25], [29], [31], and [33], respectively, and the dashed curve is the statistical-model result calculated from Eq. [27].

of $\varphi$ for the Kuwabara cell model (Case III and Case IV, which are the same with $\left.k^{*}=\left(1-C_{\mathrm{t}}^{*}\right)^{-1}\right)$ with $k^{*}$ as a parameter $\left(U / U^{(0)}\right.$ is independent of the parameter $C_{\mathrm{m}}^{*}$ in the Kuwabara model). For a fixed value of $\varphi, U / U^{(0)}$ decreases monotonically

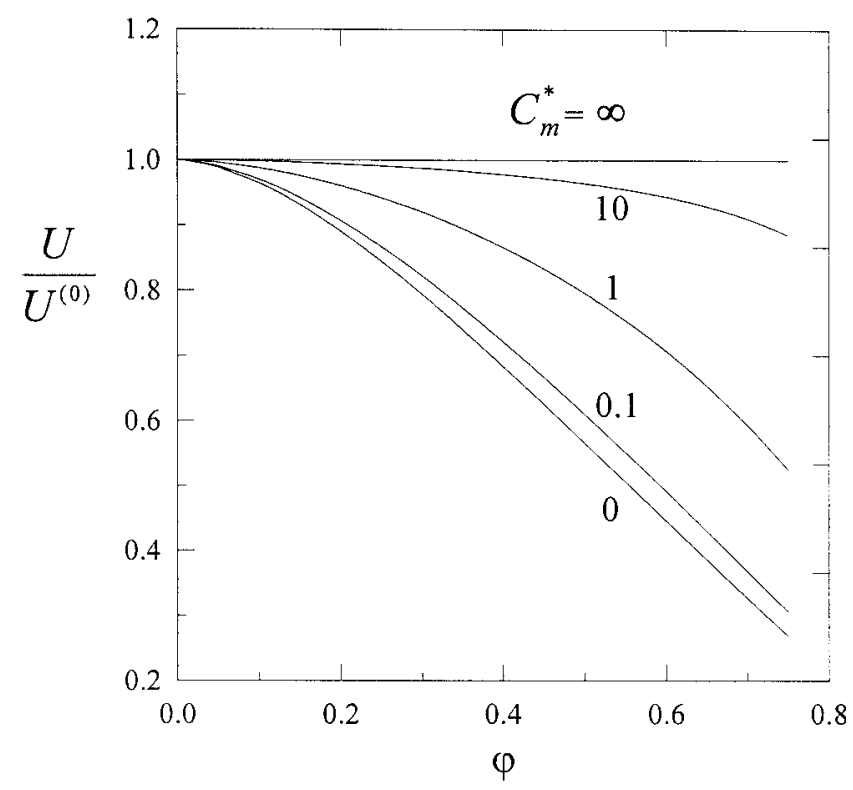

FIG . 6. Plots of the normalized thermophoretic velocity in a monodisperse suspension of spherical particles with $k^{*}=\left(1-C_{\mathrm{t}}^{*}\right)^{-1}$ versus the volume fraction of the particles with $C_{\mathrm{m}}^{*}$ as a parameter for the Happel cell model (Case I and Case II, Eq. [25] or [29]).

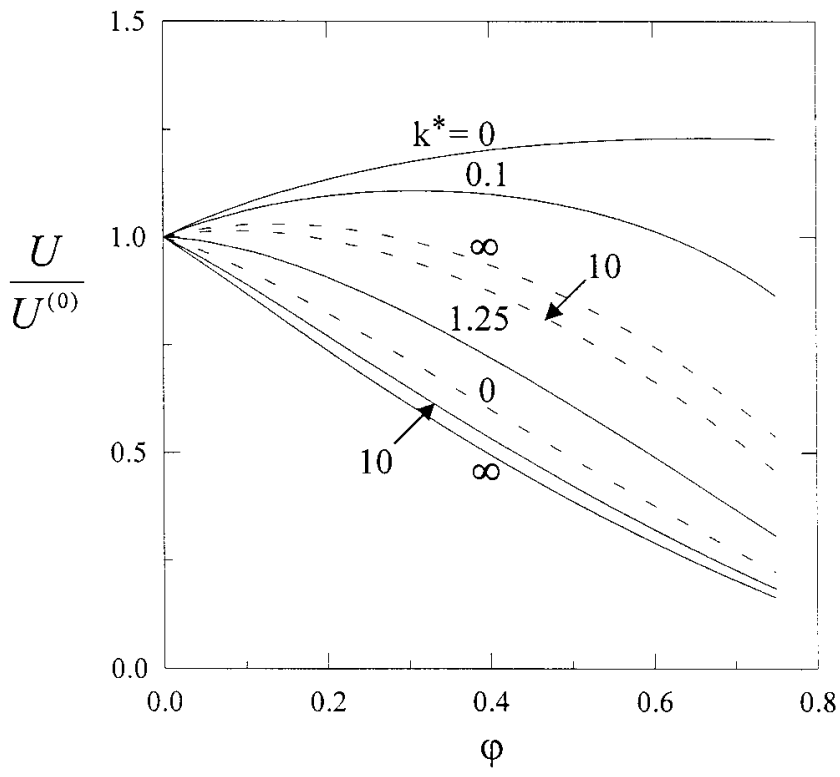

FIG. 7. Plots of the normalized thermophoretic velocity in a monodisperse suspension of spherical particles with $C_{\mathrm{t}}^{*}=2 C_{\mathrm{m}}^{*}=0.2$ versus the volume fraction of the particles with $k^{*}$ as a parameter. The solid and dashed curves represent Case I (Eq. [25]) and Case II (Eq. [29]), respectively, of the cell-model calculations.

with the increase of $k^{*}$ in Case III (opposite to the ensembleaveraged results), but increases monotonically with the increase of $k^{*}$ in Case IV. It can be seen that $U / U^{(0)}$ is not a function of $\varphi$ in the special situation of Case III with $k^{*}=0$,

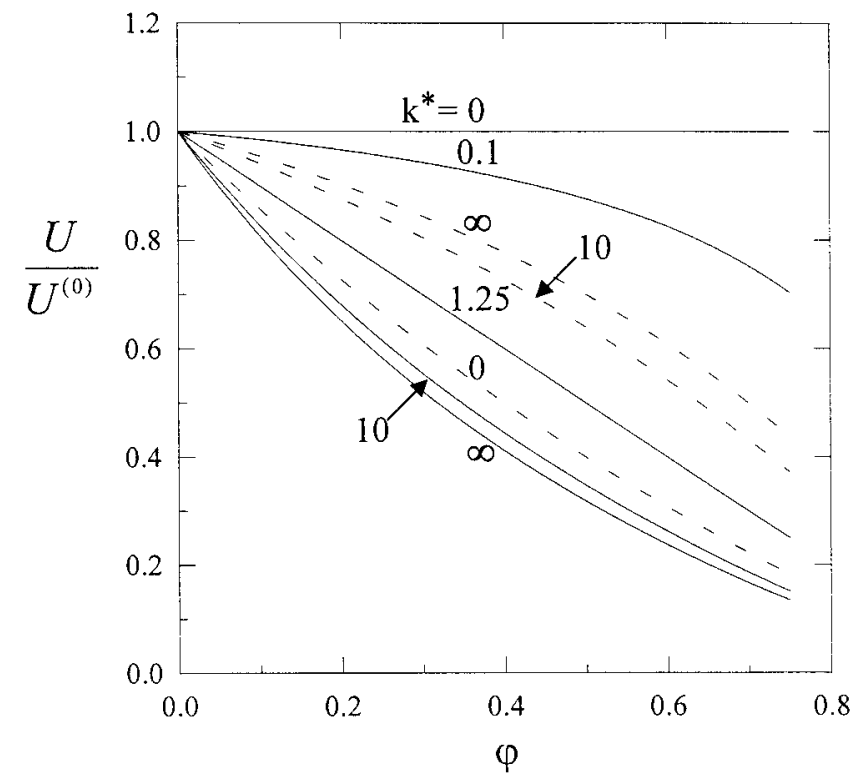

FIG. 8. Plots of the normalized thermophoretic velocity in a monodisperse suspension of spherical particles with $C_{\mathrm{t}}^{*}=0.2$ versus the volume fraction of the particles with $k^{*}$ as a parameter. The solid and dashed curves represent Case III (Eq. [31]) and Case IV (Eq. [33]), respectively, of the cell-model calculations. 
but decreases monotonically with the increase of $\varphi$ in all the other situations.

\section{CONCLUDING REMARKS}

In this paper, the thermophoresis of a swarm of identical spherical particles suspended uniformly in a gaseous medium has been analyzed using the unit cell models with various boundary conditions at the outer (virtual) surface of the cell. Each particle may have arbitrary values of its thermal conductivity and surface properties. Based on the assumption of small Knudsen, Peclet, and Reynolds numbers, the temperature and flow fields in the cell are analytically solved and the particle velocities as functions of the volume fraction of the particles are obtained in the closed-form expressions [25], [29], [31], and [33]. Comparisons of the results among the cell models and between the statistical model for dilute gas-particle systems and the cell models have also been provided.

We note that the four cases of the cell model defined in the previous section lead to somewhat different results of the particle velocity. The unit cell models with various boundary conditions at the virtual surface of the cell have also been used in the literature to study the electrophoresis of charged spherical particles, a force-free "phoretic" motion similar to the thermophoretic motion considered here. For example, Levine and Neale (25) used Case I and Case III, Zharkikh and Shilov (26) used Case II and Case IV, Kozak and Davis (27) used Case III, and Ohshima (28) used Case IV (the temperature is replaced by electric potential in these studies) to obtain the electrophoretic mobility of a well-mixed suspension of identical charged spheres. Neither of these boundary conditions is rigorously correct on the virtual surface of the unit cell, for the surrounding spheres affect the temperature (potential) and velocity at this surface. Mathematically, the temperature and flow fields in the surrounding matrix should be coupled to the corresponding fields in the unit cell through appropriate compatibility conditions (as opposed to boundary conditions), and specification of boundary conditions at the virtual surface is, at best, an approximation. Nonetheless, the analysis presented here provides meaningful information for the concentration effects on the thermophoretic mobility in concentrated suspensions of particles and for the extension of the cell-model analysis to other "phoretic" motions, such as diffusiophoresis of charged or uncharged particles $(29,30)$ and osmophoresis of vesicles (31). Our results indicate that the tendency of the dependence of the normalized particle velocity $U / U^{(0)}$ on the conductivity parameter $k^{*}$ in Case I and Case III is not correct, in comparison with the ensemble-averaged results. So, the boundary condition [4] is not as accurate as the boundary condition [7], probably due to the fact that the angular component of the temperature gradient at the virtual surface of the cell is not specified in Eq. [4]. It has been shown that the thermophoretic migration velocity predicted by Case IV of the cell model agrees quite well with that calculated from the statistical model for dilute suspensions of particles, although the Kuwabara model fails to give the dependence of $U / U^{(0)}$ on the frictional slip parameter $C_{\mathrm{m}}^{*}$. Experimental data would be needed for confirming the validity of each case of the cell model at various ranges of $k^{*}, C_{\mathrm{t}}^{*}, C_{\mathrm{m}}^{*}$, and $\varphi$.

\section{APPENDIX: SOLUTIONS FOR A SUSPENSION OF PARTICLES IN GRAVITY-DRIVEN MOTION}

In this appendix we consider the isothermal, body-force-driven motion (e.g., sedimentation) of a homogeneous distribution of identical spherical particles of radius $a$ in an incompressible Newtonian fluid, and the fluid may slip frictionally at the surface of the particle. The Reynolds number is assumed to be small. The unit cell model is used to predict the dependence of the average particle velocity $U \mathbf{e}_{z}$ on the particle volume fraction $\varphi\left(=a^{3} / b^{3}\right)$, where $b$ is the outer radius of a unit cell as shown in Fig. 1, for a given applied force $F_{\mathrm{a}} \mathbf{e}_{z}$ on each particle.

The governing equation and general solution of the stream function for the fluid flow in a cell are still provided by Eqs. [9] and [15], respectively, and the boundary conditions at the particle surface are given by Eq. [12] with $\nabla T=\mathbf{0}$. If the Happel (19) model is employed, the boundary conditions at the outer (virtual) envelope of the cell are given by Eq. [14], and the coefficients $C, D, E$, and $F$ in Eq. [15] can be expressed by Eq. [16] with $A=0$. Substitution of this coefficient $D$ into Eq. [19] leads to the drag force $F_{\mathrm{d}}\left(=-F_{\mathrm{a}}\right)$ exerted by the fluid on the particle,

$$
F_{\mathrm{d}}=-8 \pi \eta a \omega\left[3\left(1+2 C_{\mathrm{m}}^{*}\right)+2\left(1-3 C_{\mathrm{m}}^{*}\right) \varphi^{5 / 3}\right] U
$$

where $\omega$ is defined by Eq. [18]. Thus, the translational velocity of the particle can be written as

$$
\begin{aligned}
U= & U^{(0)} \frac{1+2 C_{\mathrm{m}}^{*}}{1+3 C_{\mathrm{m}}^{*}}\left[3\left(1+3 C_{\mathrm{m}}^{*}\right)-\frac{9}{2}\left(1+2 C_{\mathrm{m}}^{*}\right) \varphi^{1 / 3}\right. \\
& \left.+\frac{9}{2}\left(1-2 C_{\mathrm{m}}^{*}\right) \varphi^{5 / 3}-3\left(1-3 C_{\mathrm{m}}^{*}\right) \varphi^{2}\right] \\
& \times\left[3\left(1+2 C_{\mathrm{m}}^{*}\right)+2\left(1-3 C_{\mathrm{m}}^{*}\right) \varphi^{5 / 3}\right]^{-1}
\end{aligned}
$$

where

$$
U^{(0)}=-\left(\frac{1+3 C_{\mathrm{m}}^{*}}{1+2 C_{\mathrm{m}}^{*}}\right) \frac{F_{\mathrm{d}}}{6 \pi \eta a},
$$

which is the translational velocity of a single particle subject to an applied force $-F_{\mathrm{d}}$ in the absence of the other particles. The particle velocity $U$ can be expressed as the power expansion in $\varphi$, 


$$
\begin{aligned}
U=U^{(0)}[ & 1-\frac{3\left(1+2 C_{\mathrm{m}}^{*}\right)}{2\left(1+3 C_{\mathrm{m}}^{*}\right)} \varphi^{1 / 3} \\
& \left.+\frac{5}{6\left(1+3 C_{\mathrm{m}}^{*}\right)\left(1+2 C_{\mathrm{m}}^{*}\right)} \varphi^{5 / 3}+O\left(\varphi^{2}\right)\right]
\end{aligned}
$$

When the Kuwabara (20) model for the boundary conditions on the virtual surface of the cell (Eqs. [14a] and [21]) is used, the coefficients $C, D, E$, and $F$ in Eq. [15] can be written as Eq. [22] with $A=0$, and the drag force on the particle becomes

$$
F_{\mathrm{d}}=-120 \pi \eta a \omega^{\prime}\left(1+2 C_{\mathrm{m}}^{*}\right) U
$$

where $\omega^{\prime}$ is defined by Eq. [23]. In this case, the translational velocity of the particle can be expressed as

$$
\begin{aligned}
U=U^{(0)}\left[1-\frac{9}{5} \frac{\left(1+2 C_{\mathrm{m}}^{*}\right)}{\left(1+3 C_{\mathrm{m}}^{*}\right)} \varphi^{1 / 3}\right. & +\frac{1}{1+3 C_{\mathrm{m}}^{*}} \varphi \\
& \left.-\frac{1}{5} \frac{\left(1-3 C_{\mathrm{m}}^{*}\right)}{\left(1+3 C_{\mathrm{m}}^{*}\right)} \varphi^{2}\right]
\end{aligned}
$$

Equations [A1]-[A6] degenerate to the case of motion of no-slip particles when $C_{\mathrm{m}}^{*}=0$ and to the case of motion of gas bubbles when $C_{\mathrm{m}}^{*} \rightarrow \infty$. It can be seen in Eqs. [A4] and [A6], the leading order of the concentration effect on the particle mobility is $\varphi^{1 / 3}$, and this effect predicted by the Kuwabara model is stronger than that for the Happel model. Note that the subsequent order of the concentration effect is $\varphi$ in the Kuwabara model, but is $\varphi^{5 / 3}$ in the Happel model. Different from expressions [A4] and [A6] derived from the unit cell model, the leading order of the ensemble-averaged result for the concentration effect on the particle mobility is $\varphi$ (32).

The streamline pattern for the fluid flow around the translating particle in a cell is exhibited in Fig. A1 for the case of $C_{\mathrm{m}}^{*}=0.1$ and $a / b=0.5$. As expected, a stagnation point appears at $\theta=0$ and $z=b$. The fluid at $\theta=\pi / 2$ moves in the direction against the particle movement in the vicinity of the virtual surface of the cell, but as the fluid approaches the particle surface the streamlines become parallel to the direction of particle movement and the center of a recirculation flow appears. No qualitative difference is observed in the streamline patterns of the Happel and Kuwabara models, although the spacing between streamlines is narrower and the fluid recirculation is stronger for the Happel model, which shows a larger particle mobility.

The normalized particle mobility $U / U^{(0)}$, as calculated from Eqs. [A2] and [A6], is plotted versus the volume fraction $\varphi$ of the particles in Fig. A2 with $C_{\mathrm{m}}^{*}$ as a parameter. It can be seen that $U / U^{(0)}$ decreases monotonically with the increase of $\varphi$ for a given value of $C_{\mathrm{m}}^{*}$ and with the decrease of $C_{\mathrm{m}}^{*}$ for a fixed value of $\varphi$. For constant values of $C_{\mathrm{m}}^{*}$ and $\varphi$, the Kuwabara
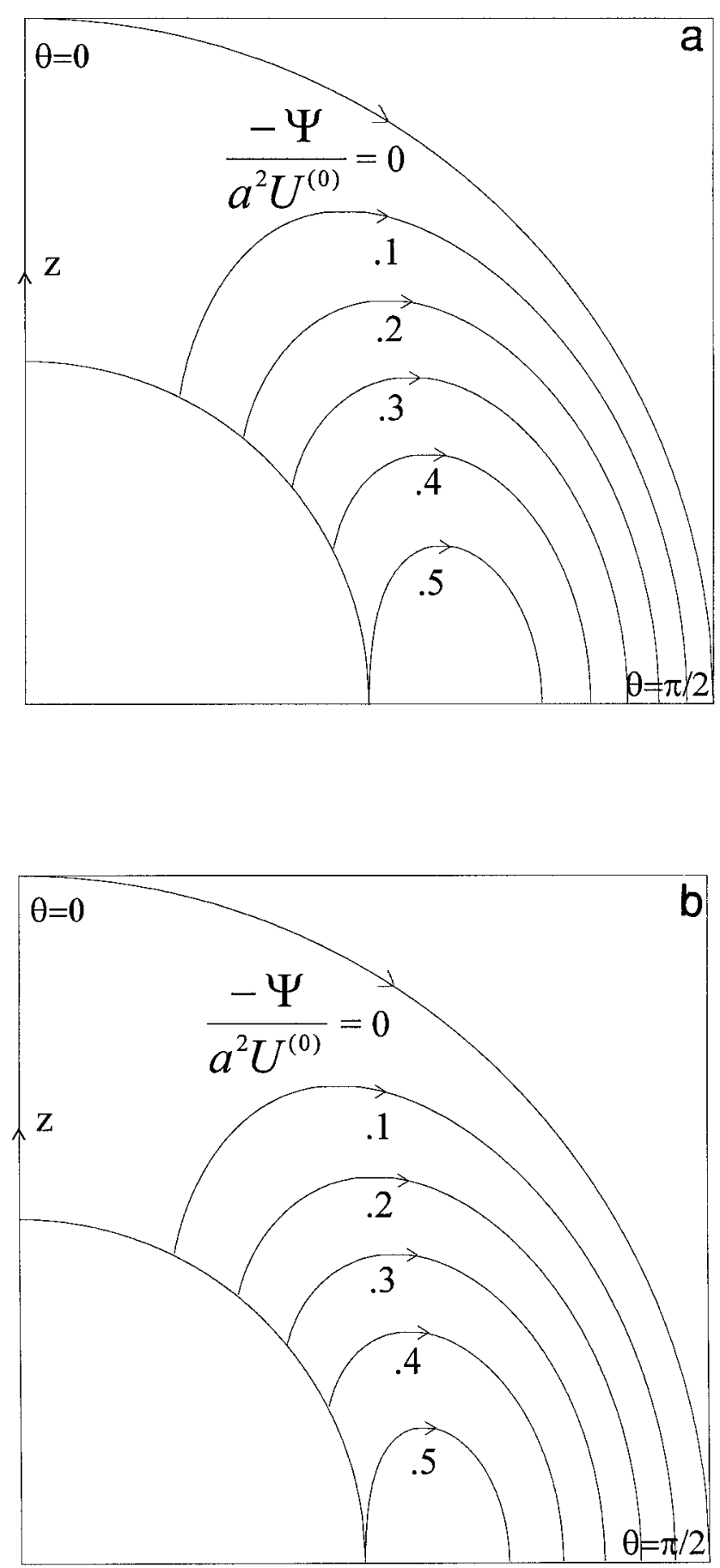

FIG. A1. Fluid streamlines for the recirculation flow around a translating spherical particle at the center of a spherical cell with $C_{\mathrm{m}}^{*}=0.1$ and $a / b=0.5$ : (a) the Happel model; (b) the Kuwabara model.

model predicts a stronger concentration effect on the particle mobility (or a smaller mean particle mobility) than the Happel model does. Note that the method of ensemble average predicts that $U / U^{(0)}=1+\gamma \varphi+O\left(\varphi^{2}\right)$ with $\gamma=-6.55$ as $C_{\mathrm{m}}^{*}=0$, $\gamma=-4.89$ as $C_{\mathrm{m}}^{*}=1$, and $\gamma=-4.49$ as $C_{\mathrm{m}}^{*} \rightarrow \infty$ (32). 


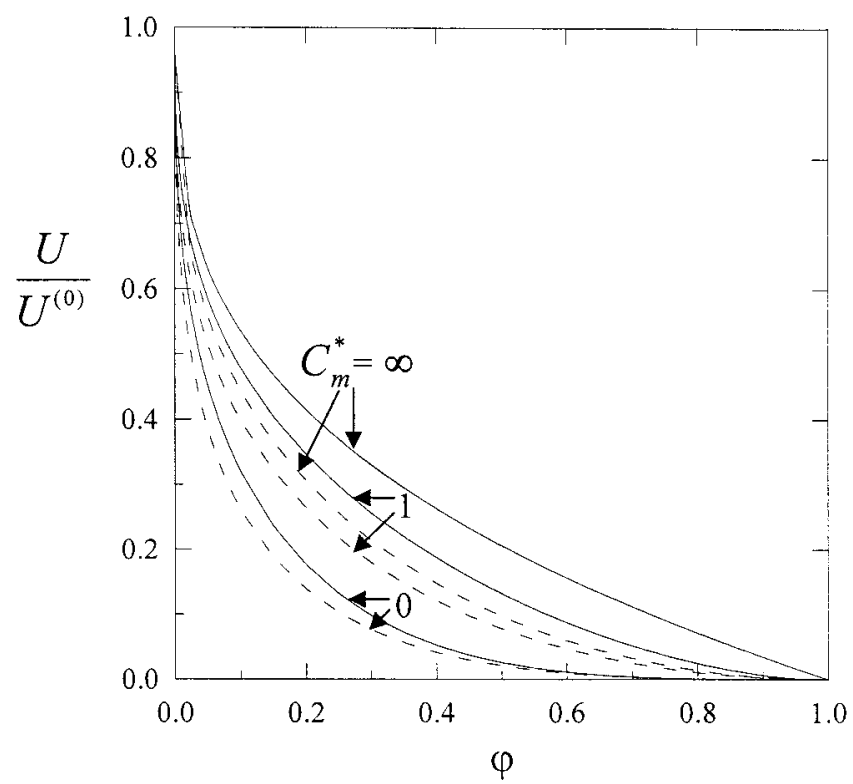

FIG. A2. Plots of the normalized sedimentation velocity in a monodisperse suspension of spherical particles versus the volume fraction of the particles with $C_{\mathrm{m}}^{*}$ as a parameter. The solid and dashed curves represent the calculations of the Happel and Kuwabara cell models, respectively.

\section{ACKNOWLEDGMENT}

This research was supported by the National Science Council of the Republic of China under Grant NSC85-2214-E002-025.

\section{REFERENCES}

1. Waldmann, L., and Schmitt, K. H., in "Aerosol Science" (C. N. Davies, Ed.). Academic Press, New York, 1966.

2. Derjaguin, B. V., Rabinovich, Ya. I., Storozhilova, A. I., and Shcherbina, G. I., J. Colloid Interface Sci. 57, 451 (1976).

3. Bakanov, S. P., Aerosol Sci. Technol. 15, 77 (1991).
4. Kennard, E. H., "Kinetic Theory of Gases." McGraw-Hill, New York, 1938.

5. Loyalka, S. K., J. Aerosol Sci. 23, 291 (1992).

6. Friedlander, S. K., "Smoke, Dust and Haze." Wiley, New York, 1977.

7. Sasse, A. G. B. M., Nazaroff, W. W., and Gadgil, A. J., Aerosol Sci. Technol. 20, 227 (1994).

8. Montassier, N., Boulaud, D., and Renoux, A., J. Aerosol Sci. 22, 677 (1991).

9. Weinberg, M. C., J. Am. Ceram. Soc. 65, 81 (1982).

10. Ye, Y., Pui, D. Y. H., Liu, B. Y. H., Opiolka, S., Blumhorst, S., and Fissan, H., J. Aerosol Sci. 22, 63 (1991).

11. Williams, M. M. R., and Loyalka, S. K., "Aerosol Science: Theory and Practice, with Special Applications to the Nuclear Industry." Pergamon Press, Oxford, 1991.

12. Brock, J. R., J. Colloid Sci. 17, 768 (1962).

13. Loyalka, S. K., Physica A 163, 813 (1990).

14. Talbot, L., Cheng, R. K., Schefer, R. W., and Willis, D. R., J. Fluid Mech. 101, 737 (1980).

15. Keh, H. J., and Chen, S. H., Chem. Eng. Sci. 50, 3395 (1995).

16. Reed, L. D., and Morrison, F. A., J. Aerosol Sci. 6, 349 (1975).

17. Chen, S. H., and Keh, H. J., J. Aerosol Sci. 26, 429 (1995).

18. Keh, H. J., and Chen, S. H., J. Aerosol Sci. 27, 1035 (1996).

19. Happel, J., AIChE J. 4, 197 (1958).

20. Kuwabara, S., J. Phys. Soc. Jpn. 14, 527 (1959).

21. Neale, G. H., and Nader, W. K., AIChE J. 20, 530 (1974).

22. Happel, J., and Brenner, H., "Low Reynolds Number Hydrodynamics." Martinus Nijhoff, The Netherlands, 1983.

23. Li, W. J., and Keh, H. J., J. Electrochem. Soc. 144, 3536 (1997).

24. Keh, H. J., and Yu, J. L., Aerosol Sci. Technol. 22, 250 (1995).

25. Levine, S., and Neale, G. H., J. Colloid Interface Sci. 47, 520 (1974).

26. Zharkikh, N. I., and Shilov, V. N., Colloid J. USSR (Engl. Transl.) 43, 865 (1982).

27. Kozak, M. W., and Davis, E. J., J. Colloid Interface Sci. 127, 497 (1989).

28. Ohshima, H., J. Colloid Interface Sci. 188, 481 (1997).

29. Anderson, J. L., Lowell, M. E., and Prieve, D. C., J. Fluid Mech. 117, 107 (1982)

30. Prieve, D. C., Anderson, J. L., Ebel, J. P., and Lowell, M. E., J. Fluid Mech. 144, 247 (1984).

31. Anderson, J. L., Phys. Fluids 26, 2871 (1983).

32. Keh, H. J., and Chen, S. H., Chem. Eng. Sci. 52, 1789 (1997). 